\title{
Ergonomic Study for Elderly Aquatic Exercises with Less Water Consumption
}

\author{
Fang-Lin Chao, Ching-Lin Lu, and Je-Ming Lin
}

\begin{abstract}
Aquatic exercises are favorite for the elderly. Buoyancy works as an upward pressure within the water in the opposite direction of gravity. It prevents compression on the joints. Peripheral providing increased resistance included pool noodle and water dumbbells for work the major muscle groups. The ergonomic study provides the design with less water consumption for the elderly. Proposed semi-sitting design reduced the water level to be $60 \mathrm{~cm}$; people can experience related water immersion exercises. This semi-sitting position included hip and back torso support to ensure safety. Elderly have less accompany and risk in this situation. The semi-sitting position designs enable some primary activities with a limited amplitude which is seniors need.
\end{abstract}

Index Terms-Ergonomic, aquatic exercises, facility design, water consumption.

\section{INTRODUCTION}

Considering healthy of the elderly, aquatic exercise has been proposed as an alternative mode of action. Aquatic activities are favorite for elder, especially who has knee joint disease. Buoyancy works as an upward pressure in the opposite direction of gravity's downward pull on the body. Exercise in water is less stressful to body joints which reducing some arthritic pain from bone on bone movement. Many factors, including chronic illness, a sedentary lifestyle, and nutritional deficiencies may contribute to muscle weakness. Shallow-water exercise programs emphasize both aerobic and resistance components. The aquatic training was helpful for women over 60 years old. The water lifting reduces the body weight and provides a muscle re-built opportunity [1]. The elderly in Japan has commonly performed the aquatic exercise. Significant improvements were observed in muscle strength in plantar flexion. Tension and anxiety in daily life were alleviated with growth of strength of the lower extremities [2].

\section{A. Design Process}

On the premise of caring for the elderly to increase their body ability, the elderly often abandon water sports because they are inconvenient to go to the swimming pool. They also worry about the risk factors in swimming pools. For example, accidental slipping may result in drowning or injury. Firstly, a personal water sports facility was developed in this study to promote exercise with less consumption of water.

As in Fig. 1, the design process of the aquatic exercise

Manuscript received September 19, 2018; revised March 1, 2018.

Fang-Lin Chao, Ching-Lin Lu, and Je-Ming Lin are with the Department of Industrial Design, Chaoyang University of Technology, Taichung, 436, Taiwan, R.O.C. (e-mail: flinchao@gmail.com). facility looks for suitable design concepts and evaluates it with a prototype. Ergonomic study refines the design to ensure safety and specific moving ability. Within a limited amount of water, the facility enables the elderly to move smoothly and take exercise with safe.

The sixteen main items in the aquatic exercise were investigated and set as the reference of evaluation. The semi-sitting posture concept was assessed with the user. Research invited the potential user to collect preliminary responses and record of a rating. The score is based on whether or not carrying out the exercise items. Finally, the fine-tuned of the design parameters were collected according to the ergonomic study.

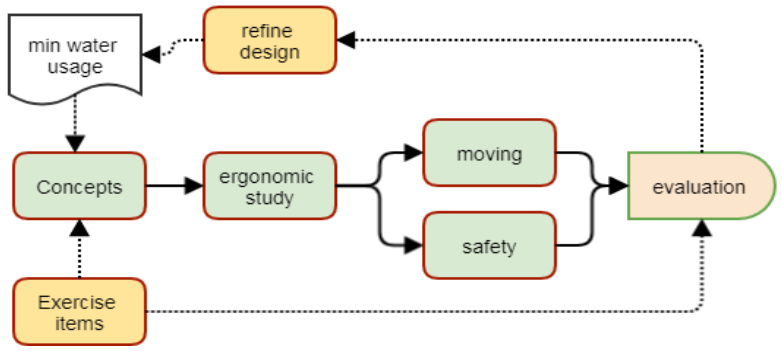

Fig. 1. The design process of the aquatic exercise facility.

\section{B. Aquatic Exercise Items}

Some aquatic program steps are listed in Table I. When the body immersed in water in a standing condition, people repeat doing the forward, backward and side movements of the legs. Considering the body balance of elderly patients, [3] observed that the aquatic exercises were significantly better for their balance than were the non-aquatic exercises. For elderly persons with cognitive impairment and dementia, exercise training increases fitness, physical function, cognitive function, and positive behavior in people. There were $81.6 \%$ improvements on flexibility, $72 \%$ on cognitive, and $72.6 \%$ on functional. The overall summarized values of exercise training improvements were $73 \%$ [3].

TABLE I: STANDARD AQUATIC EXERCISE WORKOUT ITEMS

\begin{tabular}{llll}
$\begin{array}{l}11 \text { Side leg } \\
\text { raise }\end{array}$ & 12 Leg over & 13 Rear leg lift & 14 Toe touch \\
$\begin{array}{l}21 \text { Side } \\
\text { straddle }\end{array}$ & 22 Stride hop & 23 Bounce & 24 Rise on toe \\
$\begin{array}{l}\text { 31 Side } \\
\text { bender }\end{array}$ & $\begin{array}{l}32 \text { Walking } \\
\text { craw }\end{array}$ & 33 Bouncing & $\begin{array}{l}\text { 34 Bouncing in } \\
\text { place }\end{array}$ \\
41 Knee up & 42 Twisting leg & 43 Running & 44 Push away \\
\hline
\end{tabular}

Benefits of aquatic workout elements are (1) dynamic resistance against your movements, (2) hydrostatic pressure helps with inflammation, (3) buoyancy reduce weight on knee joints. It also inherently provides resistance, creating a more effective workout than on land [4]. The benefit of aquatic 
exercises exists in elderly persons, pregnant women, and patients with osteoarthritis. Reference [5] investigated the effects of mechanical unweighting on knee pain and exercise responses in people with osteoarthritis. With a mean age of 67.9 years, mechanical unweighting treadmill exercise accompanied by BWS permits recommended training intensities obtained in seniors.

\section{Peripheral Items}

Complex movements can assist seniors by using a supporting metal frame on the surroundings. The user holds the metal frame backward with hands, lifts the leg or rotates the body while grasping the sideways. People also use peripheral items to increase resistance, such as the floating board to push forward and backward (Fig. 2). Alternatively, using a float allows the hips to float up. Lateral raises, biceps curls, rowing and chest flies can work the muscle groups of the upper body. Water dumbbells are also beneficial to cardiovascular fitness and endurance training.

The resistance device creates extra obstacle by changing the flow of water. For nursing home residents in a 10-week period, high-intensity resistance exercise training is a feasible and practical means of counteracting muscle weakness and physical frailty in seniors [6]. Seventy-nine adults, 65 years of age or older were randomly assigned to 1 of 3 groups: and walking performance was measured pre- and post of the control period. The resistance device of the aquatic exercise was effective in improving fall down risk factors in adults with arthritis [7].

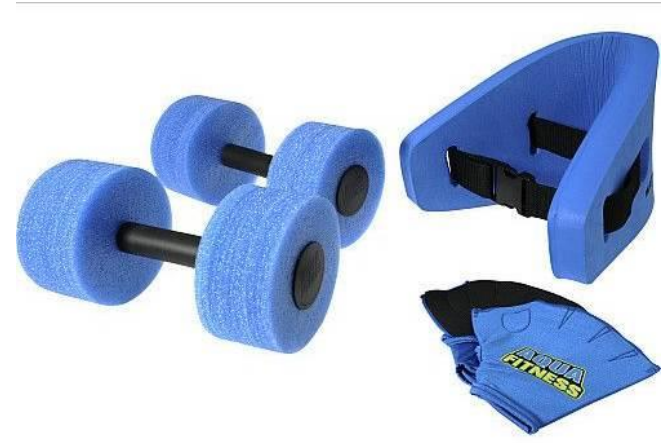

Fig. 2. Peripheral items increase resistance including pool noodles, recycled milk jugs, and water dumbbells to fit user's needs [7].

\section{Minimize Water Usage}

Water security is the availability of an acceptable quantity and quality of water for health, livelihoods. Most water-insecure countries face far more challenges today [8]. A pilot project for a new settlement in Germany demonstrated the feasibility of an integrated system with vacuum toilets and pipes for the collection of used water. Grey and stormwater collection avoided expensive settlement [9].

The amount of water usage in the swimming pool is very high. People add chlorine to maintain the water quality of the swimming pool. These chemicals have a negative influence on the human body. Excessive water consumption is a serious problem. Cherish water while attending water sports and safety of auxiliary facility are the goals of this study.

The water level required for general sports is about $160 \mathrm{~cm}$ (to the chest). If we use $60 \mathrm{~cm}$ deep of water in doing some of the water sports, we will save $2 / 3$ amount of water.

\section{E. The Safety Issue of Aquatic Exercises}

A swimming pool can be a dangerous hazard site. Taking proper steps can protect against swimming pool related accidents. A ladder is vital to prevent fall during access. A simple slip and fall can be deadly for a senior. For example, dementia elder needs special care to protect them from wandering into the pool area without proper supervision. Regardless of age or health condition, the caregivers are always required during playing.

Older adults' participation in water activity in the United Kingdom remained low [10]. The need for regular participation in PA was recognized. Sometimes, the angst of wearing a bathing costume inthe public was expressed, particularly amongst those considering they overweight. The difficulties of managing physical (e.g., injury and illness) and environmental risk were described. However, the exclusivity of many aquatic sessions emphasized the safety issue of exercise.

\section{Previous Design Proposals}

The Hydro Jogger is a portable personal exercise pool (Fig. 3 ) constructed of plastic walls to contain a body of water to perform low impact vertical and horizontal resistive exercises. Some of the attributes include automated controls and equipment management and exercise programs. The accessories constitute the complete aquatic exercise system for the home and professional clinic through adaptive convalescent therapies. The invention increases the range of movement and reduces related arthritic pain [11].
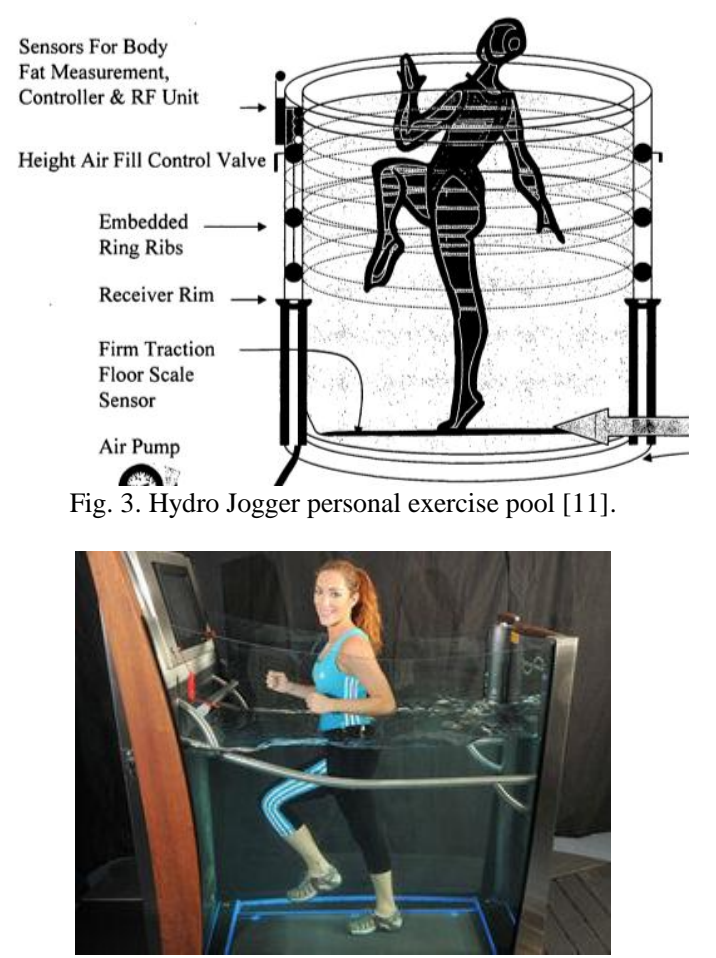

Fig. 4. The underwater treadmill using buoyancy vests in pools to help injured athletes keep their fitness levels up [12].

Fig. 4 shows injured athletes running in water to lower impact than training on the ground. The Hydro Physio underwater treadmill contained in a tank that fills up pool. One needs to wear a swimming costume and climb in the 
trainer. User response indicated: "three kilometers per hour walking is like power walking at six on land," "Because of the resistance of the water, the movement is slightly different which utilize more muscle groups," "You have to put in extra effort to stabilize." There are no signs of the normal twinges in the knee. We refer to Fig. 3 and four as the "vertical design approach."

\section{HORIZONTAL APPROACH CONCEPT}

The vertical design approach had a drawback of safety issue during the climb in and out. Considering elder's safety, we adopted the horizontal approach for the aquatic exercise design.

\section{A. Semi-sitting Concept}

The Semi-sitting concept reduces the water level to be $60 \mathrm{~cm}$ by arranging to support with semi-sitting posture (Fig. 5). People can experience water immersion and float. Some of the exercise items can still complete. The semi-sitting posture design does not mean sitting in a chair. As in Fig. 5(a) of the concept prototype, the seat, back, arm and hip support provide stability during exercise. This supporting design could interfere with some movement. But the design allows the user to try different water exercising with fewer safety concerns.

For lower-body exercises with the concept prototype, you need to be at least waist deep in the water. The lying posture in Fig. 5(b) keep waist within the water for a depth of $60 \mathrm{~cm}$.

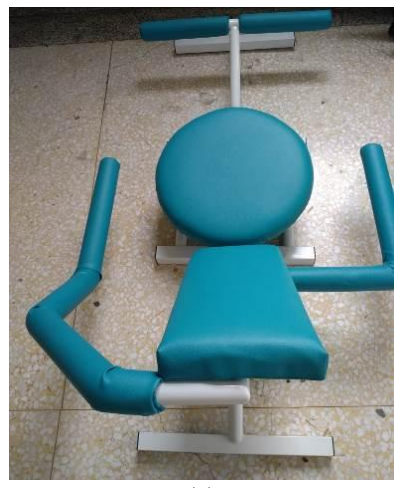

(a)

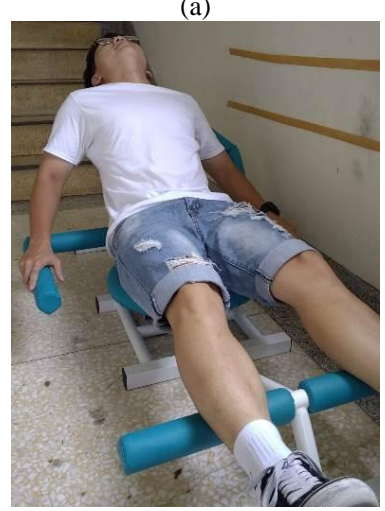

(b)

Fig. 5 Concept prototype, (a) 1:1 prototyping (b) user observation with sitting posture; the line on right-hand side wall indicate water level of $70 \mathrm{~cm}$ (upper) and $50 \mathrm{~cm}$ (lower).

\section{B. Ergonomic Study}

The dimension of semi-sitting posture design are:
- Hip support height: $18 \mathrm{~cm}$,

- Feet support height: $20 \mathrm{~cm}$,

- Hip-Feet support distance: $60 \mathrm{~cm}$,

- Armrest height (left and right): $20 \mathrm{~cm}$, length $37 \mathrm{~cm}$,

- Back torso support length: $51 \mathrm{~cm}$, skew angle 45 degrees.

For upper body movements, users prefer to see deep shoulders or neck to maximize the natural resistance of water. Try to raise the side, the primary muscle group of the upper limb, and let the biceps run to the chest by rowing. The lower body exercises required water reaching the depths of the waist. From the observation in Fig. 5, it is possible to achieve the depths of the waist with the sitting posture.

The feet support bar is helpful to the upward lower body, but it might be useless during feet movement while floating. The feet support bar extends the facility volume and weight. Trimming of feet support bar is possible for those elderly who can upward lower body by themselves. In this situation, the total length of the semi-sitting posture design can reduce the facility's length from $120 \mathrm{~cm}$ to $60 \mathrm{~cm}$.

From the user observation, an elder user needs additional support during the transition period (from sitting, exercising and standing). As in Fig. 6 and 7, it indicates the possible postures during seating, standing or exercising.

Participant need left armrest height more than $40 \mathrm{~cm}$ for pushing the body during sitting. Additional holding grab is required for stabilizing during sit-down and getting-up transition period. The posture and possible handler positions during exercise are display in Fig. 6, where holding bar can be installed. There are also holding and crawl need during the practices indicated in Table I; it will refer to future design.

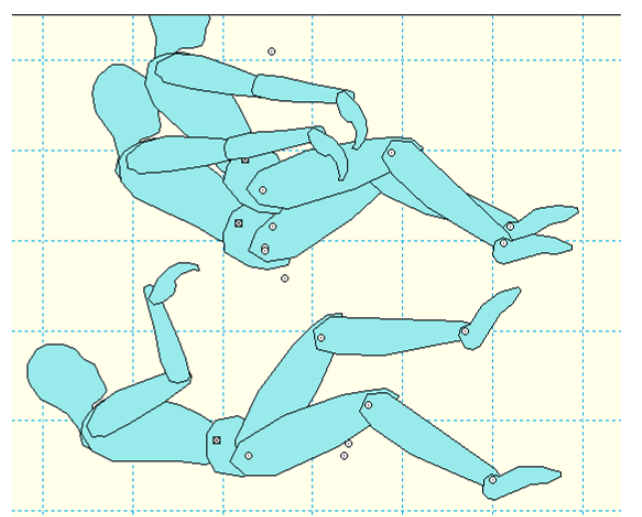

Fig. 6. Ergonomic study of seating pad and holding position (upper: sitting and standing, lower: exercising).

The concept prototype employed back torso support skew angle with 45 degrees. By user behavior observations, participants preferred the skew angle adjust to 30 degrees. The buoyancy works better with the upward pressure during floating in this situation. The back torso support majorly along the upper back can reduce size while needed. The nose position of a participant is lower than $70 \mathrm{~cm}$ water line and higher than that of a $50 \mathrm{~cm}$ water line (Fig. 7). An additional pillow can comfort the elderly with water experience.

Fig. 5 also indicates that the current concept prototype cannot fulfill the situation for the water depth of $50 \mathrm{~cm}$. As in Fig. 7, reducing the sitting height can achieve the goal of 50 $\mathrm{cm}$ water depth by removing the frame under the seat. It is possible to place an elastic pad directly on the bottom of the 
pool. The user sits in the lowest in this position. When the body is buoyant in the water, and the lower body is floating, the body can be turned and appropriately exercised.

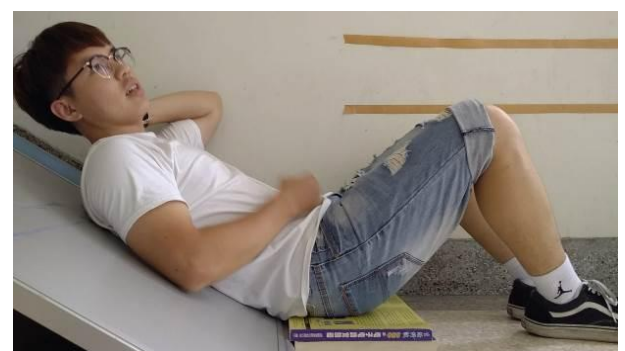

Fig. 7. Skew angle adjustment: where seat pad height is $4 \mathrm{~cm}$ (participant: Z.S Lee), where the line on the wall represents the water level of $50 \mathrm{~cm}$ and $70 \mathrm{~cm}$.

\section{Evaluations}

\section{A. The Practice of Exercise Items}

The concept Prototype as shown in Fig. 5(a) was put within the water to evaluate the possibility of doing aquatic exercise items (in Table I). The researcher requested the participant to try sixteen kinds of exercise items (Fig. 8) and record the score of user subjective feeling in Table II.

TABLE II: RECORDING SCORE OF STANDARD EXERCISE ITEMS

\begin{tabular}{llll}
\hline 11 Side leg & 12 Leg over: & 13 Rear leg lift: & 14 Toe touch: \\
raise: 1.00 & 1.00 & 0.35 & 0.70 \\
21 Side & 22 Stride hop: & 23 Bounce: & 24 Rise on toe: \\
straddle: 1.00 & 0.35 & 0.35 & 0.70 \\
31 Side & 32 Walking & 33 Bouncing: & 34 Bouncing: \\
bender: 0.70 & craw: 0.35 & 0.70 & 0.70 \\
41 Knee up: & 42 Twisting & 43 Running: & 44 Push away: \\
0.70 & leg: 0.70 & 0.35 & 0.35 \\
\hline
\end{tabular}

The recorded scores of exercise items reflect the possibility to accomplish specific exercise; where 1.00 means exercise can be done almost wholly, 0.70 means it can be done with some restrictions and 0.35 means activity with considerable limitation. Among the exercise items, three of the 16 items can be fully executed, seven items perform a limited execution, and other six items cannot be achieved.

The water volume is relatively low, and some actions cannot achieve properly. We provide support of the seat, legs, and the side handles to improve safety, they also limit the body's reaction. Therefore, there will be a trade-off between the body movement and the security in the design.

For seniors with better physical abilities, the unwanted supporting parts can be removed, such as the legs' support. The water is shallow, and there is no worry of sinking, while, it is possible to reduce the supporting elements and practice more items.

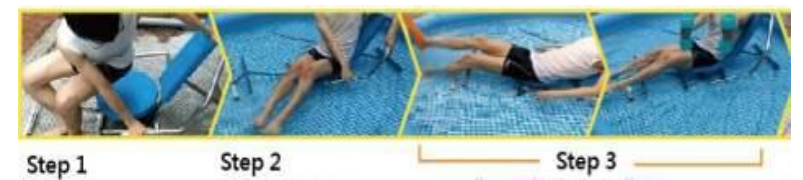

Fig. 8. The participant in doing sixteen kinds of exercise items, where the steps 1-3 represent the entire procedure from holding/ sitting/ exercises/ and standing.

\section{B. Overall Evaluations}

The overall evaluations results are shown in Fig. 9 (viewing from user accessibility, the frequency of utilizing, safety, extra care needed and total cost). In general, the sitting posture is better than normal stance. It uses less water (safety/ cost) to meet the user's needs.

The water level in the swimming pool is excellent. The swimming pool needs guidance coach and group class; it is not convenient for the user to go often. Elder regularly requests extra accompanies for the risk of slipping (sitting care and safety is better). The pick-up and drop-off problems reduced thevery access frequency. Exercising in a half-lying position extends user's population who cannot experience the buoyancy of water before.

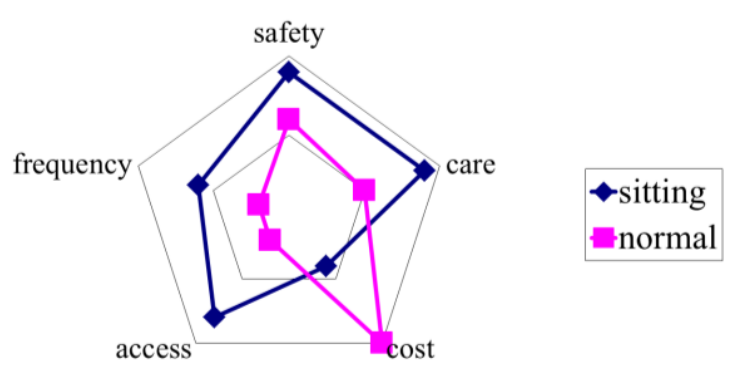

Fig. 9. Radar diagram results of overall evaluations.

\section{CONCLUSIONS}

After the reduction of water depth, the elderly can still exercise in a half-lying posture. Senior can again experience the buoyancy and resistance of water. By trimming feet supporter, the total length of the frame shrinks from $120 \mathrm{~cm}$ to $60 \mathrm{~cm}$. From the user behavior observations, they prefer 30 degrees skew angle. Based on the ergonomic study, we found that sitting and standing is essential. We can trim the length and size of back torso supporting to let buoyancy and exercise freely works. Immerse timmershe body in water with the water depth of $50 \mathrm{~cm}$ is possible. A $5 \mathrm{~cm}$ thickness elastic pad can reduce the sitting heightpad, sitting and standing are required additional help for the people.

\section{REFERENCES}

[1] T. Tsourlou, A. Benik, K. Dipla, A. Zafeiridis, and S. Kellis, "The effects of a twenty-four-week aquatic training program on muscular strength performance in healthy elderly women," Journal of Strength and Conditioning Research, vol. 20, no. 4, p. 811, 2006.

[2] Y. Katsura, T. Yoshikawa, S. Y. Ueda et al., "Effects of aquatic exercise training using water-resistance equipment in elderly," European journal of applied physiology, vol. 108, no. 5, pp. 957-964, 2010.

[3] N. C. Avelar, A. C. Bastone, M. A. Alcântara, and W. F. Gomes, "Effectiveness of aquatic and non-aquatic lower limb muscle endurance training in the static and dynamic balance of elderly people," Brazilian Journal of Physical Therapy, vol. 14, no. 3, pp. 229-236, 2010.

[4] T. M. Barbosa, M. F. Garrido, and J. Bragada, "Physiological adaptations to head-out aquatic exercises with different levels of body immersion," Journal of Strength and Conditioning Research, vol. 21, no. 4, p. 1255, 2007.

[5] K. K. Mangion, K. Axen, and F. Haas, "Mechanical unweighting effects on treadmill exercise and pain in elderly people with osteoarthritis of the knee," Physical therapy, vol. 76, no. 4, pp. 387-394, 1996.

[6] S. M. Resende and C. M. Rassi, "Effects of hydrotherapy in balance and prevention of falls among elderly women," Brazilian Journal of Physical Therapy, vol. 12, no. 1, pp. 57-63, 2008.

[7] C. M. Arnold andR. A. Faulkner, "The effect of aquatic exercise and education on lowering fall risk in older adults with hip osteoarthritis," 
Journal of Aging and Physical Activity, vol.18, no. 3, pp. 245-260, 2010.

[8] D. Grey and C.W. Sadoff, "Sink or swim? Water security for growth and development," Water Policy, vol. 9, no. 6, pp. 545-571, 2007.

[9] R. Otterpohl, M. Grottker, and J. Lange, "Sustainable water and waste management in urban areas," Water Science and Technology, vol. 35, no. 9, pp.121-133.

[10] A. B. Evans and M. Sleap, "You feel like people are looking at you and laughing, older adults' perceptions of aquatic physical activity," Journal of Aging Studies, vol. 26, no. 4, pp. 515-526, 2012.

[11] B. Bullman and R. Walker et al., "Complete and portable aquatic exercise system called: The Hydro Jogger," U.S. Patent Application, p. 324, 2005.

[12] Hydro Physio. [Online]. Available: https://www.hydrophysio.com/

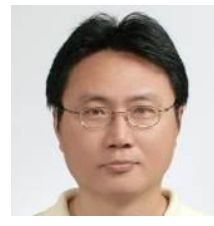

Fang Lin Chao was born in Taipei, Taiwan, ROC, in 1958. He received the Ph.D. degree in electrical engineering from National Taiwan University, in 1991.

He joined the Electronic Research and Service Organization, Industrial Technology Research Institute, Hsinchu, Taiwan, in 1982. His responsibilities included thermal, structural, and electrical modeling for the system. In 1994, he was an associate professor with the Department of Industrial Design at Da-Yeh Institute of Technology, Taiwan. Since September 2004, he has been an associate professor with the Department of Industrial Design at Chaoyang University of Technology, Taichung, Taiwan. His research interests include the electronic packaging design, eco-design, and design integration in product development. 